\title{
Corporate Governance, Management Control and Global Competition
}

\author{
Daniela M. Salvioni*
}

\begin{abstract}
Corporate governance is a complex activity evolving in parallel with change within internal and external contexts. In particular, global competition shows how maintaining the conditions for company effectiveness implies: reviewing the corporate governance approach, emphasising relations between governance and management control and developing appropriate skills to adapt to the variables being monitored.

The new outlook companies are up against tends to determine revising important critical factors for company success and changes resources important for the creation and maintenance of positive company/environment relations. More specifically, certain elements are taking shape that - though with different characteristics and importance based on specific company contexts and interaction with the environment - are proving to be dominant success factors. These include: corporate culture; information system; brand equity.
\end{abstract}

Keywords: Corporate Governance; Management Control; Critical Success Factors; Intangible Assets

\section{Corporate Governance and Global Competition}

Changes over the last decade within the competitive context companies have to deal with, have led to profound changes in corporate governance and in related management control systems ${ }^{1}$.

Market and information globalisation, frequent over-supply situations for goods, growing complexity in intra and inter company relations, the importance of innovation for products and processes, the need to recover faith in and consensus in relation to company operations all highlight the importance of an integrated approach to governance processes and the need to improve direct models and define how effective the many types of operating units are.

\footnotetext{
* Full Professor of Business Administration, University of Brescia (salvioni@eco.unibs.it)
}

Edited by: ISTEI - University of Milan-Bicocca

ISSN: 1593-0319

Salvioni Daniela M., Corporate Governance, Management Control and Global Competition, Symphonya. Emerging Issues in Management (www.unimib.it/symphonya), n. 1, 2005, pp. 39-51

http://dx.doi.org/10.4468/2005.1.03salvioni 
Eliminating existing space and time barriers has highlighted the need to adopt a global corporate governance view, starting from the top, involving the whole organisation and coordinating all behaviour. In such an environment, corporate governance and internal control systems are distinguished through organisational structures, targets and various processes with a common objective aimed at optimising company performance.

At present, companies have to take greater care than previously over external relations, societal expectations, management orientation, organisational behaviour and results.

Company success is tied to the ability to move down governance paths based on: appreciation of stakeholders expectations, rational translation of expectations into strategies, transferring of management orientation to general behaviour, constant control over consistence between targets, management objectives and results actually achieved. For this, attributing value to the control support role takes on considerable importance for the effective implementation of governance decisions, with integration between corporate governance and management control and with the latter becoming part of a unitary internal control system.

Management control as a basic process for implementing governance actions consequently highlights the limits and inadequacy of a limited view of control, closely centred round quantitative-financial measures and formal control or mainly on appreciation of operating efficiency conditions.

Management control is that critical aspect for maximising company effectiveness, as it helps to obtain results in line with expectations through: centralised coordination, hierarchical development of targets and assigning targets to basic internal organisation units, controlling consistency between targets and results, interpreting any differences between targets and results, and finally, preparing appropriate reports (management reporting).

The developments recently suffered by economic systems have, however, highlighted the need to improve company-environment relations, specifically:

- the need for more responsible governance, in line with a multidimensional view, aimed at fairly matching legal, economic, social and environmental aspects;

- the opportunity to adapt management control systems to ensure consistency between governance choices, organisation behaviour and actual results.

Corporate governance is thus a complex activity evolving in parallel with change within internal and external contexts. In particular, global competition shows how maintaining the conditions for company effectiveness implies: reviewing the corporate governance approach, emphasising relations between governance and management control and developing appropriate skills to adapt to the variables being monitored (Figure 1). 
Figure 1: Impact of New Outlooks on Governance and Control

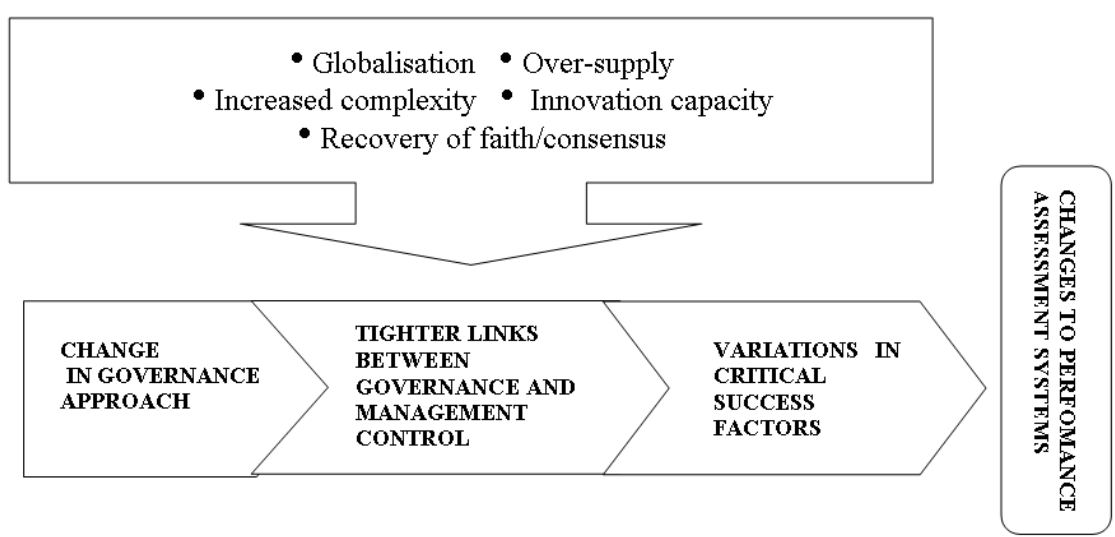

Indeed, management control must recast itself as corporate governance tries to fully acquire all those variables needed for company success. At the same time, governance must know how to exploit effective management control process opportunities that are part of the global internal control systems (internal control in a strict sense, internal auditing and management control) and that react in a tight relationship to cost/benefit logic.

\section{Global Market and Corporate Governance}

In global markets, corporate governance objectives are progressively expanding as governance becomes involved in the whole internal and external network, in an approach based on satisfying expectations and exchanging information ${ }^{2}$.

Corporate governance decisions used to be taken with respect to shareholders interest (shareholder view) and there were often conflicts between governance bodies and managers. That kind of behaviour exhibited limits in terms of lasting development potential.

Changes brought on by global competition and growing exposure and permeability to information flow with the various societal players, has highlighted the importance of relations between company success conditions and a fair approach to the mix of stakeholders expectations. In that sense, a view of company responsibilities as a whole over space and time is consolidating, replacing the focus on short-term economic-financial results - an expression of a partial approach to giving majority relevance to satisfying those providing risk capital.

Corporate governance is therefore evolving from a situation of attention paid to shareholders and related economic responsibility to giving clear value to all stakeholders and company liabilities as a whole (legal/administrative, economic, social and environmental) (Figure 2).

The passage from a shareholder view to a stakeholder view asserts the role of independent administrators ${ }^{3}$ and is supported by the spread of control processes (internal and external), codes and standards ensuring behavioural propriety and equity. Thus, over recent years, the evolving competitive context has imposed a governance concept by which: 
- only competitive companies, able to respond to market needs and generate value, show ample and not fleeting success potential and significant sustainable development prospects;

- effective interaction with all stakeholder groups influences the ability to obtain consensus and suitable resources to sustain company development, and affects the optimisation of economic results and company value.

Figure 2: Effectiveness Conditions for Corporate Governance

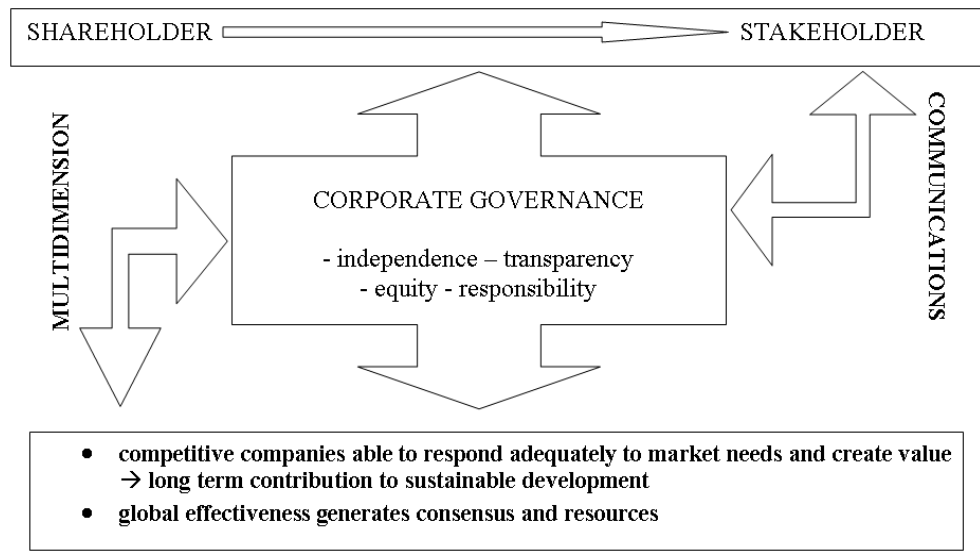

The context outlined shows frequent changes to standards, recommendations and discipline codes aimed at regulating governance structures and inter-company relations, but also at deeply modifying governance direction and related management control processes.

Global markets impose the spread of an equity, propriety and transparency culture and emphasises the careful setting up of links between all societal player expectations, governance decisions and management activities. For this, just refer to the recent introduction of managerial positions and behaviour codes aimed at creating a common, shared approach towards company problems (e.g. the spread of ethical codes, the implementation of plans to construct an ethical company culture and introduction of those responsible for ethics, social responsibility managers and Ethical Committees). All this together with frequent re-defining of project control mechanism variables consistent with internal culture and relative permeability.

Thus the maintenance of satisfying company effectiveness levels is related to the will to promote adequate learning and cultural growth. This is in addition to a company's ability to initiate governance processes developed along an ideal line, from the top, involving the entire organisation and orienting behaviour in a coordinated manner.

In the absence of an important influence between governance structure decisions, environmental position, management phenomena and organisation behaviour orientation, governance activity risks losing a lot of its usefulness. Situations of decisional responsibility could occur with no correspondence in actual management behaviour. Hence the importance of management control transferring governance decisions to organisation behaviour, through a coordination and behaviour orientation function that optimises success and permanence in relation to the various, changing internal and external phenomena. 
The main management control role lies in effective support action for governance bodies to get management results consistent with governance expectations. Furthermore, control (with its processes) helps producing culture, generates learning and, above all, gives governance bodies updated, thorough assessment elements on threats and opportunities existing in the various operating departments. Control helps governance, and governance is in turn an essential presupposition for creating valid management control systems.

Alongside this new interest in company governance can be found a growing need that has become evident to adapt internal control systems and make management control more flexible.

Control, as stated previously, suffers from the new outlooks companies operate in, and highlights the tighter links with corporate governance and the need to review assessment parameters. This is closely related to the changes to suitable critical factors defining economic, social and competitive success conditions.

\section{Control System Development}

Corporate governance is based on control activities with both propriety (administrative and procedural) and risk monitoring functions, with behaviour orientation against general objectives and organisational coordination and analysis of results against expectations.

Corporate governance processes are supported by internal control systems, set up to: supply all important decision elements; give validity to the intermediation function between stakeholder expectations and management behaviour; ensure compliance with laws and internal regulations; ensure completeness and transparency of communications from management, and externally.

In the present competitive context, control activity supporting governance is somewhat complex, as it requires intervention in various directions and uses different distinct but tightly related structures and processes. For that, we use the term control systems meaning all tools and procedures used to help optimise performance.

Internal control systems highlight, in turn, several distinct sub-systems related to: diversification of dedicated resources; organizational positioning and autonomy; type of intervention and relative consistency; the need for a clear separation between those controlled and controller ${ }^{4}$. In this situation, management control naturally has a main role, that is the togetherness of structures and processes aimed at assisting the proper execution of management decisions, orienting all organisational behaviour and at checking consistency between targets and results effectively achieved.

As evidence of this, given the political nature of management implementing company effectiveness and considering the cause/effect relations between method chosen to satisfy stakeholders, strategic targets, operational targets, and effective results, the link between corporate governance and responsible management development is intuitive.

Management control effectiveness is related to implementation of chosen strategic and operational targets and to minimising risks. It requires internal 
penetration and continuity and presupposes the ability to adapt to changes in management policy and the environment.

Management control evolves alongside changing industrial systems and production, organisational, information, competition and market variables. For that, recent change in the competitive context has undoubtedly emphasised the roles of management control and company effectiveness, while modifying critical factors and observation parameters (Figure 3).

Figure 3: The Management Control Role in Corporate Governance

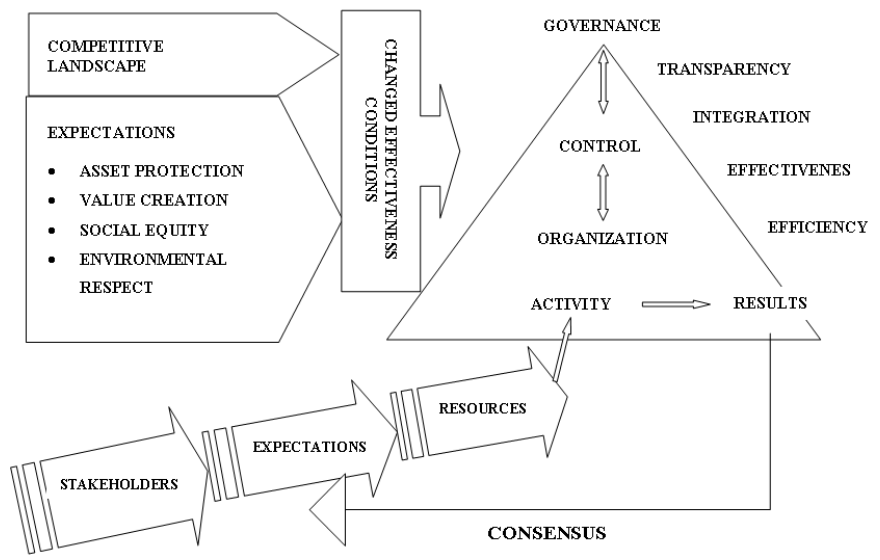

For the revamped management control role over company effectiveness, important change stimulus is certainly due to developments in market conditions and to global competition phenomena.

The gradual typical company market passage from scarcity situations to balance between supply and demand, to recent over-supply situations has influenced corporate governance processes and related control activities ${ }^{5}$. This is a slow development with distant origins and manifests itself at different speeds in differing national contexts, tending to show characteristics that are very similar at world level following the recent fall in pre-existing space and time barriers.

The increase in significant expectations for the forming of success presuppositions and the evolving competitive outlook have produced considerable changes in company effectiveness conditions and in the related ways of implementing them. In particular: the assertion of the stakeholders view has placed economic, competitive and social success at the same level; the weight of customers and suppliers amongst important stakeholders has increased; the importance given to achieving production results satisfying market expectations has grown; intermediation between governance choices and organisation behaviour - typical of management control - has changed profoundly.

Without going into all the variations occurring to maintain high quality control systems - compared to the development of various operational areas and the need to adapt to complex internal and external factors - the impact that globalisation and changes in the demand-supply relationship have had on these systems is discussed below. More specifically, the main control aspects in scarcity economies and oversupplied markets have been considered. 


\subsection{Control in Scarcity Situations}

When companies had no trouble in selling products as long as they could satisfy (and/or promote) specific needs in line with reference context characteristics (in general spatially limited), control systems mainly focused attention on operational efficiency given the limits placed by governance choices and external dynamism. The limited amount of information available, the lengthy times and high costs to obtain it tended to limit the range of action for the various operating units and reduced speed of change in competitive strategy.

In this situation, once the strategic development lines had been defined and the company product/market combinations chosen, performance optimisation and the creation of competitive advantage was mainly associated with the capacity to contain costs. This was related to the use of production factors needed to obtain goods of a certain pre-defined quality. In said conditions, control was used in budgeting, determining and analysing costs and differences between planned and effective values; standardisation processes centred on objective efficiency levels were not rare and profit was the main economic lever for extensive accountability.

In particular, recurring close relations between the various operating units, concentration on well defined, stable product/market combinations, market conditions and demand/supply relations, high information costs tended: on the one hand, to create noticeable governance deformity in various sized companies with various capital raising methods; on the other, to highlight the critical role of operational efficiency, predefined cost structure, the long-term production capacity saturation level and behaviour aimed at containing costs of the various short-term production factors. Thus the confusion (both theoretically and practically) between control system and budgeting and analytical accounting ones, as well as between administrative systems used to make control operational and the information needed to control properly ${ }^{6}$.

\subsection{Control in Over-Supply Conditions}

The move from scarcity to over-supply has given growing importance to the ability to innovate, to anticipate customer expectation changes, to create distinct, qualifying elements for the company offering. At the same time the stakeholders become important (e.g. customers and suppliers), there a need for production flexibility, geographical extension of activities, and fast and low cost information circulation.

The pre-existing time and space barriers tend to disappear; there is tighter integration between governance and control; and control systems have to implement a drastic change in how they work. In particular, critical success factors are more and more complex; there is a need to balance effectiveness and management efficiency and a need for flexible adjustment to the various production and market contexts the company has to refer to simultaneously.

Competitive changes also determine moving from strict focusing on tangible factors (production factors, products, activities, etc.) and giving higher value to intangible assets and resources.

Furthermore, the multi-dimensional governance approach means improving/refining pre-existing economic measures and selective introduction of new measurements. Alongside traditional economic-financial indicators there is growing space for indexes 
monitoring significant non-financial quality and quantity variables and constructive integration between information collected and processed in the various company areas. For this, the financial factor does not lose its importance but links between economic variables and non-economic factors increase jointly instrumental in acquiring consensus and trust.

The new value given to ethics and transparency highlights the control role thus ensuring proper transfer of governance orientation to the organisation, closely related to planning, remuneration, career and incentives, spreading an ethics culture, information and communication.

Progress made by information allows for valid control systems even if the various operating units and markets are spread all over the world, helping the capacity to differentiate, anticipate and adjust to the change in customer expectations and the spread of accountability centred round results.

Finally, increased company complexity, the need to ensure equity, transparency, propriety in governance processes, increased awareness of the importance of risk management are just some of the phenomena leading to creation of internal integrated control systems strongly based on considerations of good value and collaboration. In this context, the need to coordinate and integrate management control increases; it is specifically responsible for behaviour in implementing corporate governance trends.

The need to maintain top-level competitiveness in global, over-supplied markets leads to changing behaviour and monitoring processes considerably to maintain the effectiveness of the whole organisation related to governance trends. In particular, critical company success factors are changing and management control has to adjust both its framework information system and the way it carries out processes, accounting and integration with other forms of internal control supporting governance to said changes.

\section{Variables Monitoring}

Besides being related to proper planning of structural components, management control quality is related to the ability to single out success factors and translate them into constantly updated target parameters; that is into elements able to sum up what determines effectiveness and efficiency for governance implementation.

The elements influencing company development and relative order of priorities recommend to analyse: how the various inter-company relations, governance choices, critical resources and related attraction ability and risk state are made up; the typical activity, connected relations between phenomena and related dynamism factors; market and co-makership relations; customer/user consensus and the potential to create competitive advantage situations in various action areas; strong and weak points in relations set up in essential resource supply areas; internal structural components, organisational structure, management style and culture dominating human resources; the amount of information needed and how the information is spread to manage internal and external consensus.

Environmental change, the spread of ICT, the reduced difficulty in accessing information for all operators and changes to how you obtain consensus, as was said, have caused: a deep change in the different stakeholder roles, highlighting how the satisfaction of each group is through fair consideration of all interests and developing 
special attention for communication processes; progressive transformation of critical success factors with strong implications both on relations with the environment and corporate governance, and in terms of important management control variables.

In particular, market and information globalisation, at the end of the last century, highlighted some changes on how to conceive assumptions for lasting company success, first of all due to (Figure 4$)^{7}$ :

Figure 4: Variations to Critical Success Factors

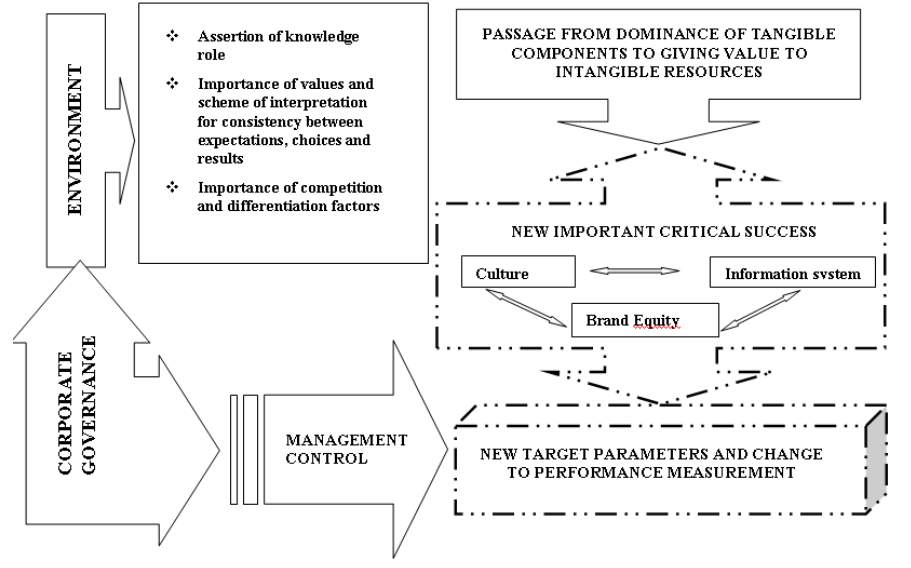

a) Assertion of knowledge as an important element in appreciating expectations, to set up suitable decision-making processes - both governance and management for coordination between decisions and actions and for the creation of positive relations with all stakeholder groups;

b) Growing importance of competition to satisfy all expectations and the importance, in this area, of anticipating customer preference changes, creating stable distinctive elements to help qualify the company offering and in measuring the customer satisfaction level and the ability to create competitive advantage factors;

c) Importance of considering dominant values, attitudes and interpretation models shared both internally and in relations between governance bodies and organisation, and in the various external interaction areas, to help create consistency between choices, activities, results and expectations.

The elements mentioned highlight an incredible change in the main critical success factors, going from a distinct dominance of tangible components qualifying the various productive combinations to a situation with value given to specific intangible components on which the forming of competitive, economic and social effectiveness assumptions can depend.

Thus, the changed context widens the critical factors to be monitored, alongside giving value to integration between governance and management, with increased need for internal transparency and in inter-company relations, and increased importance of effectiveness as a behaviour condition. At the same time, the importance given to results with regard to important factors (tangible or intangible) imposes studying new 
target-parameters, relative enhanced flexibility based on how fast one can redefine company position and selectively adjusting performance measurement.

The global competitive context emphasises how critical it is to be able to anticipate changes in customer expectations often spread widely geographically and with behaviour influenced by various cultural and economic values.

Furthermore, comparing goods that are really different seems to be coming more common (especially in more advanced contexts) so the same amount of money can be used indiscriminately for purchasing goods that are very different from one other. In this kind of situation, the importance of being able to innovate increases, production becomes flexible, there are noticeable reductions in product life cycles and relations between customer expectations (in economic terms: need satisfaction, ease of supply terms, etc.) and goods availability takes on specific importance.

The new outlook companies are up against tends to determine revising important critical factors for company success and changes resources important for the creation and maintenance of positive company/environment relations. More specifically, certain elements are taking shape that - though with different characteristics and importance based on specific company contexts and interaction with the environment - are proving to be dominant success factors. These include: company culture; information system; brand equity.

The intangible resources mentioned have specific features making control useful but complex, such as:

- they are substantially based on knowledge and on an accumulation process;

- relative value has an internal function due to governance and management decisions sensitive to creating stable competitive advantages;

- they are highly volatile so have to be fed continuously otherwise investments done over periods can be cancelled fast;

- they cannot be negotiated (purchased/sold) if not in special situations as their relative value depends on the context they were created in;

- they influence each other and are inter-dependent for the creation of stable competitive, economic and social advantage elements;

- they are conditioned by consensus management choices and by communication processes started and received by the company;

- they cannot represent directly within the company economic measurement system though often such factors can noticeably condition the creation of stable riches;

- the relative value influences a number of other intangible management elements (which we can call second level or derived) such as competitive positioning, effectiveness of product and process development, perceived quality of product/service, adequacy of operating mechanisms, suitability of behaviour and internal motivation, quality of external relations and so on.

In global, over-supplied markets, these intangible factors are an important source of wealth that has to be given adequate value and monitored to ensure proper success potential. So we must: define development lines and initiate governance processes (corporate and management) centred round singling out target parameters symptomatic of relative value growth; integrate giving value to intangible resources when analysing company asset growth (tangible and intangible). 
Controlling intangible assets and resources adds control variables that complete the traditional ones.

Companies, at least the most advanced, seem to have understood the growing importance of intangible assets. At present, we are still far from the assertion of effective, complete models to control intangible factors, especially due to the difficulty in singling out parameters that can allow monitoring of importance, flexibility, measurability, clarity and selectivity ${ }^{8}$.

Thus the control system has new challenges, specifically oriented towards abandoning an almost exclusive focus on financial measures in favour of a composed, integrated togetherness of parametric quantities directly, or indirectly, aimed at evaluating company performances.

The evolving competitive landscape highlights the importance of the related network between internal and external variables and between quality and quantity. This is even more obvious if we consider intangible resources that, for their nature, have their main answer in quality parameters; the difficulty found in control consists in the ability to translate quality elements into structured, consistent quantity indicators. For this, indirect measures (financial and non-financial) have taken on greater importance; orientated to also considering the vast influence the mix of various intangible company factors has on global results, on organisation and on strategic activity area effectiveness.

Recent studies on intangible corporate resources have analysed the choice of target parameters for control, singling out first and second level parameters ${ }^{9}$. As an example, here are some control parameters that can be used to monitor intangible corporate resources.

Company culture control parameters can be based on ethical and entrepreneurial values and include: at a first level, elements such as quality of inter-organisation relations, accountability level, transparency in relations and communication, the ability to find creative solutions, growth in professional competences, coordination difficulty, sharing and participation, consistency between resources and results, respect of others and trust, honesty, opening towards innovation; at a second level, one can go into further detail with analysis of quantity elements like how often differences between targets and results appear, how fast important information and risk factor indications are transmitted internally, the number of training requests, productivity indicators, frequency of actions taken to improve respect of ethical codes, and so on.

For the corporate information system, at a first level, importance lies in indicators such as how it answers knowledge needs, the ability to perceive changes in customer expectations, indication of risks and opportunities, how it answers communication needs; at a second level, we can use indicators such as number of integration requests, degree of information reliability, frequency of inconsistency between information need and availability, time to adjust to changes, how long it takes to get useful data for decision making and assessment needs, how often the information produced is consulted, relations between information available and information used, etc.

Finally, brand equity finds determinants internally and externally such as: at a first level, going back to quality of internal processes and to the spread of a sense of belonging, brand awareness, satisfaction of intermediate and end demand, financial institutional confidence, stakeholder consensus; at a second level, variables such as sales and market share trends, frequency of complaints about behaviour and products, number of visits to the company web site, launch time for a new product, time lapse 
between request for and obtaining financial resources, frequency of product and process adapting to market and competitor changes, the ratio costs/results for promotion activities, and so on.

Thus, the change to the competitive outlook has modified critical company factors, target parameters and, consequently, induced important changes in the measurement systems used to ensure control effectiveness. In particular, traditional economic measurements have been progressively replaced by an integrated measurement system, aimed at adapting quantity-financial processing and at combining financial with customer satisfaction results, internal productivity, learning, cultural growth and satisfaction of each important stakeholder group. Several surveys and research studies have moved in this direction and have suggested new economic processing types (for example activity-based costing, life-cycle costing, target costing, redesign to cost, etc) ${ }^{10}$, plus widening and integrating different measurements (for example, value-based management, balanced score card, internal and external benchmark analysis, etc. $)^{11}$.

\section{Notes}

${ }^{1}$ This contribution is related to the author's report presented on 10 May 2005 at the convention on 'Corporate governance between law changes and changing company models', held in Rome at Luiss-Libera Università Internazionale degli Studi Sociali Guido Carli.

2 'Corporate governance represents the relationship among stakeholders that is used to determine and control the strategic direction and performance of organisation'... 'Corporate governance has been emphasised in recent years because some observers believe that corporate governance mechanisms have failed to adequately monitor and control top-level managers' strategic decisions', M.A Hitt, R.D. Ireland and R.E. Hoskinsson, Strategic Management Competitiveness and globalisation, South-Western College Publishing Cincinnati, 2001, p. 402 and p. 403.

${ }^{3}$ For example, in Italy, the Self-discipline Code for quoted companies (issued in 1999 and then reviewed in 2002) establishes that a suitable number of non-executive directors must be independent, that is:

a) Not have, directly, indirectly or for third parties, nor have had recently, economic relations with the company, with its subsidiaries, with executive directors, the shareholder or group of shareholders controlling the company, to an extent that could condition judgement;

b) Not own, directly or indirectly, or for third parties, shareholdings for an amount that could allow them to exercise considerable control or influence over the company, nor be part of para-social pacts for company control;

c) Not close relations of executive company directors or of subjects indicated in the above points a) and b);

${ }^{4}$ For more detailed analysis of internal control systems please refer to: D.M.Salvioni, Il sistema di governo aziendale, in D.M.Salvioni (ed.), Corporate governance e sistemi di controllo della gestione aziendale, Franco Angeli, Milan, 2004.

${ }^{5}$ For this please refer to: J.J. Lambin, S.M. Brondoni, Ouverture de 'Market-Driven Management', Symphonya. Emerging Issues in Management (www.unimib.it/symphonya), n. 2, 2000-2001.

${ }^{6}$ This phenomenon is still widespread in Italy. Just think that the Anglo-Saxon texts on managerial accounting are often translated by the title planning and control.

${ }^{7}$ Please refer to: D.M. Salvioni (ed.), Corporate governance, controllo di gestione e risorse immateriali, Franco Angeli, Milan, 2004.

${ }^{8}$ Please refer to: G.Gandini, Il sistema delle risorse immateriali, in D.M. Salvioni (ed.) Corporate governance, controllo di gestione e risorse immateriali, Franco Angeli, Milan, 2004. 
${ }^{9}$ For this, please refer to contributions by S. Franzoni, F.Gennari and A. Mazzoleni, D.M. Salvioni (ed.) Corporate governance, controllo di gestione e risorse immateriali, Franco Angeli, Milan, 2004.

${ }^{10}$ Please refer, amongst others, to: R.S. Kaplan and R. Cooper, Cost \& Effect: Using Integrated Cost Systems to Drive Profitability and Performance, Harvard Business School Press, Boston, 1998.

${ }^{11}$ Please refer to: J.M. stern, J. Shiely, I. Ross, The EVA Challenge. Implementing Value-Added Change in an Organisation, John Wiley \& Sons, New York, 2001; R.S. Kaplan and D.P. Norton, The Strategy Focused Organisation, Harvard Business School Press, Boston, 2000. 